\title{
All Technologies Work Together for Good: A Glance to Future Mobile Networks
}

\author{
Animesh Yadav, Member, IEEE, and Octavia A. Dobre Senior Member, IEEE
}

\begin{abstract}
The astounding capacity requirements of 5G have motivated researchers to investigate the feasibility of many potential technologies, such as massive multiple-input multipleoutput, millimeter wave, full-duplex, non-orthogonal multiple access, carrier aggregation, cognitive radio, and network ultradensification. The benefits and challenges of these technologies have been thoroughly studied either individually or in a combination of two or three. It is not clear, however, whether all potential technologies operating together lead to fulfilling the requirements posed by 5G. This paper explores the potential benefits and challenges when all technologies coexist in an ultra-dense cellular environment. The sum rate of the network is investigated with respect to the increase in the number of small-cells and results show the capacity gains achieved by the coexistence.
\end{abstract}

Index Terms-5G, ultra-densification, massive MIMO, mmWave, small cells, full-duplex, NOMA.

\section{INTRODUCTION}

$\mathbf{I}$ $\mathrm{N}$ the last decade, the advancements in the very-largescale integrated-circuit electronics have led to an advent of affordable smart handheld wireless devices, which are now within reach of millions of people. These devices are commonly used for Internet-based applications, such as online banking, e-commerce, social media, multimedia streaming, and online gaming besides the voice application. For Internetbased services, each device needs to connect to a wireless network. As a result, a high number of subscribers straightforwardly translates into high Internet data traffic in the wireless network. Additionally, the billions of Internet-of-Things (IoT) devices, with applications to smart healthcare, smart homes and cities, smart electricity grids, etc., are expected to use the wireless network. According to Ericsson's technical mobility report published in 2017, around 29 billion connected devices are forecast by 2022 , of which around 18 billions will be related to IoT. Hence, it is quite evident that one of the key challenges in future wireless networks is the unprecedented high volume of data traffic.

Towards accomplishing the anticipated humongous volume of data traffic among other requirements, telecom industry leaders, standardization agencies, and academia are working on solutions for upcoming fifth generation $(5 \mathrm{G})$ of wireless network. Particularly, the third generation partnership project (3GPP) has initiated the 5G New Radio (NR) standardization process. The 5G NR is not just about achieving 1000 times

This work has been supported in part by the Natural Sciences and Engineering Research Council of Canada (NSERC), through its Discovery program.

Animesh Yadav and Octavia A. Dobre are with the Faculty of Engineering and Applied Science, Memorial University, St. John's, NL, A1B 3X5, Canada (e-mail: \{animeshy, odobre\}@mun.ca). higher network spectral efficiency (SE), but also about providing ubiquitous connectivity, guaranteed quality-of-service (QoS), low-cost and massive machine-type communication (mMTC), 100 times better energy efficiency (EE), support for $500 \mathrm{~km} / \mathrm{h}$ mobility, ultra-reliable low-latency communication, and increased battery operating lifetime of devices [1]

This article discusses solutions that have the capability of delivering the extreme capacity demand of the $5 \mathrm{G}$ and beyond networks. Broadly, techniques for capacity enhancement of a network can be classified into four classes: i) use of additional wider bandwidth; ii) spectrum reuse; iii) use of new technologies; iv) efficient spectrum management. Within these classes, several potential technologies have been identified to achieve the goals setup for 5G networks. Among them, the ultradense network (UDenseNet) of small-cells (SCs), full-duplex (FD) transceivers, millimeter wave (mmWave) communications, massive multiple-input multiple-output (mMIMO), nonorthogonal multiple access (NOMA), and dynamic spectrum access (DSA) technologies are the primary ones in achieving the goal of large capacity.

Individually, each technology has great potential benefits. Some of these technologies complement each other when they are combined to work together, and can achieve higher performance gains compared to their individual performances. For example, the joint use of mmWave and mMIMO technologies provides tangible capacity enhancement [2]. The highly directional beams are desirable for mmWave communications, which can be obtained by using mMIMO. Additionally, smallwavelengths at mmWave frequencies help in realizing smallsize antennas, which are essential for mMIMO deployment. Nevertheless, combining all these technologies is not trivial, and poses additional challenges. In this article, we study the benefits and challenges of simultaneously operating these technologies, and bring the following contributions:

- A comprehensive overview of the key enabling technologies for $5 \mathrm{G}$ and beyond wireless networks is provided and their fundamental features, advantages and disadvantages are introduced;

- The state-of-the-art coexistence of two or three technologies is reviewed and a novel coexistence of all key technologies is envisaged for throughput enhancement in future wireless networks. Open challenges related to the coexistence are also discussed;

- The impact of the number of SCs on the sum rate in coexistence scenarios is quantitatively studied using numerical simulations, and insights are presented.

The rest of this article is organized as follows. In Section II, we summarize the fundamental features, benefits and chal- 
TABLE I

A BRIEF SUMMARY OF VARIOUS KEY ENABLING TECHNOLOGIES FOR 5G AND BEYOND.

\begin{tabular}{|c|c|c|c|}
\hline Technologies & Fundamental features & Advantages & Disadvantages \\
\hline UDenseNets & $\begin{array}{l}\text { Amorphous deployment of } \\
\text { hundreds of SCs in a macro-cell } \\
\text { region, i.e., small ratio between } \\
\text { the number of SCs and users }\end{array}$ & $\begin{array}{l}\text { Large coverage, low power, high } \\
\text { throughput, enables spatial frequency reuse }\end{array}$ & Inter-cell CCI \\
\hline mmWave and VLC & Large amount of bandwidth & $\begin{array}{c}\text { Both provide high SE; } \\
\text { mmWave: enables the use of a large } \\
\text { number of miniature-size antennas; } \\
\text { VLC: low-cost infrastructure, i.e., LEDs } \\
\text { and inherited link security }\end{array}$ & $\begin{array}{l}\text { mmWave: high signal attenuation, } \\
\text { short transmission distance; } \\
\text { VLC: low-modulation bandwidth } \\
\text { and inter-symbol interference }\end{array}$ \\
\hline mMIMO & $\begin{array}{l}\text { Use of hundreds of antennas for } \\
\text { communication }\end{array}$ & $\begin{array}{l}\text { Surplus degrees of freedom including } \\
\text { higher SE and EE, low-power transmission, } \\
\text { simple linear signal processing }\end{array}$ & $\begin{array}{l}\text { Requires a large number of RF } \\
\text { chains, as well as estimation of a } \\
\text { large number of CSI coefficients }\end{array}$ \\
\hline FD & $\begin{array}{l}\text { Transmit and receive on the same } \\
\text { time and frequency resource }\end{array}$ & $\begin{array}{c}\text { Theoretically doubles the SE without } \\
\text { additional spectrum requirement }\end{array}$ & Self-interference and CCI \\
\hline NOMA & $\begin{array}{l}\text { Multiplex several users on the } \\
\text { same time and frequency resource }\end{array}$ & $\begin{array}{l}\text { Enhances SE without additional spectrum } \\
\text { requirement, better user fairness }\end{array}$ & Multiuser interference \\
\hline DSA & Utilization of underused spectrum & Improves spectrum utilization efficiency & $\begin{array}{l}\text { Interference from secondary to } \\
\text { primary users or networks }\end{array}$ \\
\hline
\end{tabular}

lenges of key technologies. In Section III, we discuss the feasibility of the coexistence of these technologies for 5G and beyond networks. Open research issues are introduced in Section IV. In Section V, we present numerical simulation results for an UDenseNet, where such technologies work together. Finally, the paper is concluded in Section VI.

\section{Key EnAbling TeChnologies For 5G Networks AND BEYOND}

This section provides an overview of technologies that play a pivotal role for emerging $5 \mathrm{G}$ and beyond wireless networks. A brief summary of their fundamental features, advantages, and disadvantages is presented in Table I.

\section{A. Network Ultra-Densification}

The concept of cell densification has evolved from 4-5 macro-cell base stations (MBSs) $/ \mathrm{km}^{2}$ in the third generation wireless networks to about $8-10$ micro-cell BSs $/ \mathrm{km}^{2}$ in the fourth generation. The primary aim of cell densification is to address the problem of capacity and coverage by spatial frequency-reusing and offloading the data traffic to the SCs [3]. The macro-cells in the fourth generation have a smaller area than those in the third generation. Further, SCs bring users closer to the BS, which reduces the access distance, and consequently, the path loss between them. In 5G, the SC area would be reduced further to support low-power transmissions, and hence, the cell density is expected to increase to 4050 small-cell BSs (SBSs) $/ \mathrm{km}^{2}$. However, the UDenseNets increase the interference power levels, and consequently, the overall network performance might degrade due to inter-cell co-channel interference (CCI). Hence, interference mitigation is paramount for a network to achieve high SE.

\section{B. Millimeter-Wave and Optical Wireless Technologies}

It is evident that current wireless cellular networks are unable to cater the future throughput demand, as the existing frequency bands are limited and congested. A straightforward approach is to explore higher frequencies, which offer increased bandwidth. To this end, mmWave bands ranging from 30-300 GHz, have been investigated for cellular purposes [4]. At such frequencies, the main technical challenge is the severe attenuation due to path loss, shadowing, and blockage, along with the high energy consumption. The directional beams are used to combat the path loss and other losses.

Another promising approach is the optical wireless communication (OWC), which enables the use of large bandwidths in optical bands such as infrared, visible light, and ultraviolet spectra. In particular, the visible light spectrum band ranging from $400-800 \mathrm{THz}$ can be used freely for cellular purposes. The visible light communication (VLC) exhibits inherited network security, and it is easier to integrate using existing lightening infrastructure, e.g., light emitting diodes (LEDs). Since the visible light does not penetrate through opaque materials, VLC is a suitable candidate for indoor environments. The main challenges in VLC are the lowmodulation bandwidth of LEDs, inter-symbol interference and CCI.

\section{Massive MIMO Technology}

The MIMO technology has been well recognized for enhancing the SE, EE, and reliability of a wireless link. Due to the use of multiple antennas at both transmitter and receiver sides, a MIMO system provides multiplexing and diversity gains. In a multi-user case, MIMO enables communication of multiple users simultaneously on the same radio resource. On the other hand, by significantly increasing the number of antennas to tens or hundreds, i.e., mMIMO system, the $\mathrm{SE}$ and EE can be significantly improved beyond those of the traditional MIMO systems. Other advantages associated with mMIMO are: simplified signal processing, such as zeroforcing transmitter and maximum ratio combining receiver; channel hardening effect; and low-power transmission due to high beamforming and array gains [5]. The major difficulty in deploying mMIMO is the requirement of a large number of radio-frequency $(\mathrm{RF})$ chains and a large number of channel state information (CSI) coefficient acquisition, as well as 
pilot contamination. A combination of digital and analog beamformers is an efficient solution to reduce the number of RF chains. Various efficient hybrid beamforming structures are discussed in [6].

\section{Full-Duplex Technology}

Recently, the FD technology has become an active research area because of its ability to double the SE of a network without requiring new spectrum. Hence, the FD technology has the potential to contribute towards meeting the high capacity and low-latency $5 \mathrm{G}$ requirements. However, an FD transceiver needs to cope with self-interference (SI), which suppresses the low-power received signal by the high-power transmitted signal. Several works have focused on developing efficient SI cancellation techniques. The experimental results show that by applying a combination of passive and active cancellation methods, SI can be made as low as the thermal noise level. The active methods include analog cancellation at the RF stage and digital cancellation at the baseband stage. Another performance limiting factor is the CCI between the downlink (DL) and uplink (UL) users, which can be reduced by employing optimal power allocation and scheduling schemes.

\section{E. Non-Orthogonal Multiple Access}

Likewise FD technology, NOMA is another promising costeffective technique that improves the SE of a network without the need of additional bandwidth resources. Furthermore, it also ensures user fairness, which is an important metric from the perspective of network operators. In NOMA, multiple users are multiplexed mainly in code- or power-domain and transmit over the same frequency and time resource. For example, sparse code multiple access is a code-domain NOMA scheme, where the codewords of different users are superimposed nonorthogonally using sparse spreading, and multiuser detection and data recovery are jointly performed at the receiver side. On the other hand, in power-domain NOMA DL transmission with two users, a BS superimposes the user data and allocates more transmit power to the user with worse channel conditions. Each user then performs the successive interference cancellation (SuIC) to decode the information by eliminating the undesired interference [7]. On one hand, on a DL channel, NOMA outperforms the traditional orthogonal multiple access (OMA) scheme, in terms of both sum rate and fairness. On the other hand, when compared to the non-linear dirty-paper coding, NOMA has lower computational complexity; however, at the cost of some performance loss.

It is worth mentioning that UL NOMA can support massive connectivity, which is highly desirable for mMTC scenarios. Furthermore, in a grant-free UL access, latency reduction could be expected due to control signaling minimization.

\section{F. Dynamic Spectrum Access}

The previously discussed technologies yield increased SE; however, they do not solve the problem of spectrum underutilization. The DSA technology provides a promising way that enables the use of underutilized frequency bands [8]. The
DSA techniques broadly fall into two categories: i) cognitiveinspired radio access, which allows the secondary networks to use temporarily or spatially unoccupied bands without harmful interference to the primary networks; ii) cooperative-inspired radio access, where the primary networks allow spectrum sharing, trading, and leasing to the secondary networks with some economic favor. For example, the DSA technology plays a key role in the seamless integration of the ad-hoc deviceto-device (D2D) communications into the bandwidth scarcenetwork. The D2D links are supposed to provide low-latency, low-power, and high peak data rate service between two network users.

\section{A Future Cellular Network Design}

This section discusses the future cellular network design, where all key enabling technologies coexist. A summary of a few state-of-the-art technologies that are combined with a perspective of improving the overall network throughput is given in Table II.

\section{A. Coexistence of Key Enabling Technologies}

In addition to the cellular traffic, the next generation wireless network supports traffic from D2D and IoT communications as well. Hence, seamless coexistence of all key technologies is pivotal to achieve a large throughput in the 5G and beyond wireless networks. Moreover, from the perspective of the infrastructure of a macro-cell belonging to a fogcomputing-based radio access network (F-RAN) architecture, a significant difference that the upcoming future networks would bring is the massive deployment of several low-cost and low-power SBSs. Several fixed and mobile relays, as well as fog-computing-based BSs (F-BSs) and a large number of remote radio heads (RRHs) will be used for an enhanced performance. The F-BSs are fixed low-power BSs, which are connected to the core network either directly or through the pool of baseband units (BBUs) or through the MBS. The F-BS's connections to the BBU and MBS are performed using fronthaul links and optical fibers, respectively. The MBS and RRHs are connected to the BBUs for baseband processing via $\mathrm{X} 2$ interface and fronthaul, respectively. The F-BSs have extra signal processing functionalities to perform some tasks, e.g., cooperative radio signal processing and interference management, locally rather than centrally at the BBU. Local processing helps in reducing latency and burden on the fronthaul and BBU. A graphical representation of a macro-cell infrastructure and links is depicted in Fig. 1. For a seamless coverage, users closer to SBSs and RRHs are served by SBSs and RRHs, respectively.

To better understand the potential benefits and challenges posed by the co-existence of all key enabling technologies, we divide the discussion into wireless access and backhauling in the SC networks.

\section{B. Wireless Access in the SCs Network}

1) UDenseNets, mmWave and OWC: The amorphous and ultra-dense deployment of SCs reduces the access distance 
TABLE II

A BRIEF SUMMARY OF THE STATE-OF-THE-ART COMBINATION OF VARIOUS KEY ENABLING TECHNOLOGIES FOR 5G AND BEYOND.

\begin{tabular}{|c|c|c|c|}
\hline Combinations & Coupling factors & Problems & Possible solutions \\
\hline $\begin{array}{c}\text { UDenseNets and mmWave } \\
\qquad 9]\end{array}$ & $\begin{array}{l}\text { Large network throughput and mmWave signals } \\
\text { travel short distance due to large propagation loss; } \\
\text { hence, they are a good fit for UDenseNets }\end{array}$ & $\begin{array}{l}\text { In an urban environments, mmWave } \\
\text { suffers further propagation loss due to } \\
\text { shadowing and blockage; hence, it } \\
\text { requires highly directional beams for } \\
\text { transmission }\end{array}$ & mMIMO \\
\hline mmWave and mMIMO & $\begin{array}{l}\text { mMIMO helps in achieving the much needed } \\
\text { highly directed beam for mmWave operation, and } \\
\text { the small wavelengths of mmWave help } \\
\text { accommodating a large number of miniature-size } \\
\text { antennas in a small space }\end{array}$ & $\begin{array}{l}\text { The digital beamforming architecture } \\
\text { for mMIMO requires a large number } \\
\text { of RF chains corresponding to each } \\
\text { antenna, and unaffordable energy } \\
\text { consumption }\end{array}$ & $\begin{array}{l}\text { Hybrid beamforming } \\
\text { architecture and } \\
\text { beamspace MIMO }\end{array}$ \\
\hline mMIMO and NOMA 10 & $\begin{array}{l}\text { Especially, beamspace MIMO supports one user per } \\
\text { RF chain, which limits the number of users that the } \\
\text { SBS can support. Integrating beamspace MIMO } \\
\text { with NOMA technique eliminates this limitation }\end{array}$ & $\begin{array}{l}\text { Cannot support large number of users } \\
\text { due to the decoding complexity of } \\
\text { SuIC, which scales with the number of } \\
\text { users, as well as due to the problem of } \\
\text { error propagation in SuIC [11] }\end{array}$ & $\begin{array}{l}\text { Group a small } \\
\text { number of users per } \\
\text { RF chain and apply } \\
\text { FD communication } \\
\text { for each chain }\end{array}$ \\
\hline $\begin{array}{l}\text { mMIMO and FD } 12] \\
\text { NOMA and FD } 13]\end{array}$ & $\begin{array}{c}\text { The highly directional beams generated by mMIMO } \\
\text { help reducing the LOS SI at the FD transceiver; } \\
\text { NOMA and FD together improve SE. NOMA } \\
\text { improves user fairness }\end{array}$ & $\begin{array}{l}\text { Huge surge of intra- and inter-cell CCI } \\
\text { due to FD communication }\end{array}$ & $\begin{array}{l}\text { Efficient resource } \\
\text { management and } \\
\text { interference } \\
\text { mitigation techniques }\end{array}$ \\
\hline
\end{tabular}

between the users and SBS, and provides an unlimited user experience. The SCs can be deployed in both indoor and outdoor environments. However, using the existing cellular bands for communication in these cells should be avoided as they travel far beyond the cell boundaries and cause severe inter-cell CCI. The mmWave bands are a good alternative as the signals travel shorter distance because of the large propagation loss, and hence, generate low inter-cell CCI. Moreover, for an indoor case, the spectrum from the optical bands is an option as well. For instance, in a room or a corridor of a building or a house, there can be a VLC-SC that serves several users without creating inter-cell CCI. Hence, the mmWave and OWC technologies complement well with the network densification [9]. In an outdoor urban environment, mmWave is a viable option; however, the users in a mmWaveSCs achieve smaller signal-to-interference plus noise ratio due to the blockage effect.

2) UDenseNets, mmWave, OWC and mMIMO: In order to improve the data rate performance of the mmWave-SC users and cope with the large propagation loss, the mmWave signal needs to be highly directional, which can be achieved either by using a large number of antennas or directional antennas. The small wavelength at mmWave frequencies allows packing a massive amount of miniature-size antennas to form an antenna array for transceivers. Additionally, the massive amount of antennas not only boosts up the received signal power via array gain, but also enhances the SE of the network using multiuser MIMO techniques.

However, the mmWave and mMIMO integration requires a large number of RF chains that accounts for approximately $70 \%$ of the total transceiver energy consumption [5]. Hence, this particular integration is economically prohibitive, and energy and cost-efficient solutions have been explored. A fully analog and hybrid (analog-digital) beamforming architectures have been investigated. The fully analog architecture requires only one RF chain with an analog circuit for adjusting the phases of signals. As such, this architecture supports a single transmit beam, which makes difficult to adjust the beam to the channel conditions, and results in considerable performance loss. On the other hand, the hybrid beamforming architecture supports a minimum number of RF chains, which helps in reaping the benefits of multiple antennas, and offers a reasonable trade-off between cost and performance [6]. A different alternative solution which has recently surfaced is the beamspace MIMO technique; this significantly reduces both the number of required RF chains and the energy consumption [14]. However, in the beamspace MIMO, each RF chain supports only one user at the same time-frequency resource, which is its fundamental limitation. Thus, the number of users supported by this scheme cannot exceed the number of RF chains.

3) UDenseNets, mmWave, OWC, mMIMO, and NOMA: When further integrating NOMA with mmWave and mMIMO technologies, the limit of one user per RF chain can be overcome through the multiplexing capability of NOMA. However, in DL power-domain NOMA, at the user end, the decoding complexity of SuIC scales with the number of users [11], [15]. Therefore, a smaller number of users, generally two users, are grouped per RF chain.

4) UDenseNets, mmWave, OWC, $m M I M O$, NOMA, and FD: Furthermore, by allowing each SBS to operate in FD mode, an RF chain can serve users both in the DL and UL channels, which results in higher SE. Theoretically, FD communication doubles the SE of the network when there is zero SI and CCI. As previously mentioned, the mmWave technology enables the formation of antenna arrays by using massive miniature-size antenna elements that are arranged very close to each other. This arrangement leads to poor passive isolation between the transmit and receive antennas and causes higher SI. Nevertheless, the beamforming achieved with the antenna array helps to overcome the problem of passive isolation. Moreover, highly directional signals have only low-power reflected non line-ofsight (NLOS) components that contribute towards the SI. 


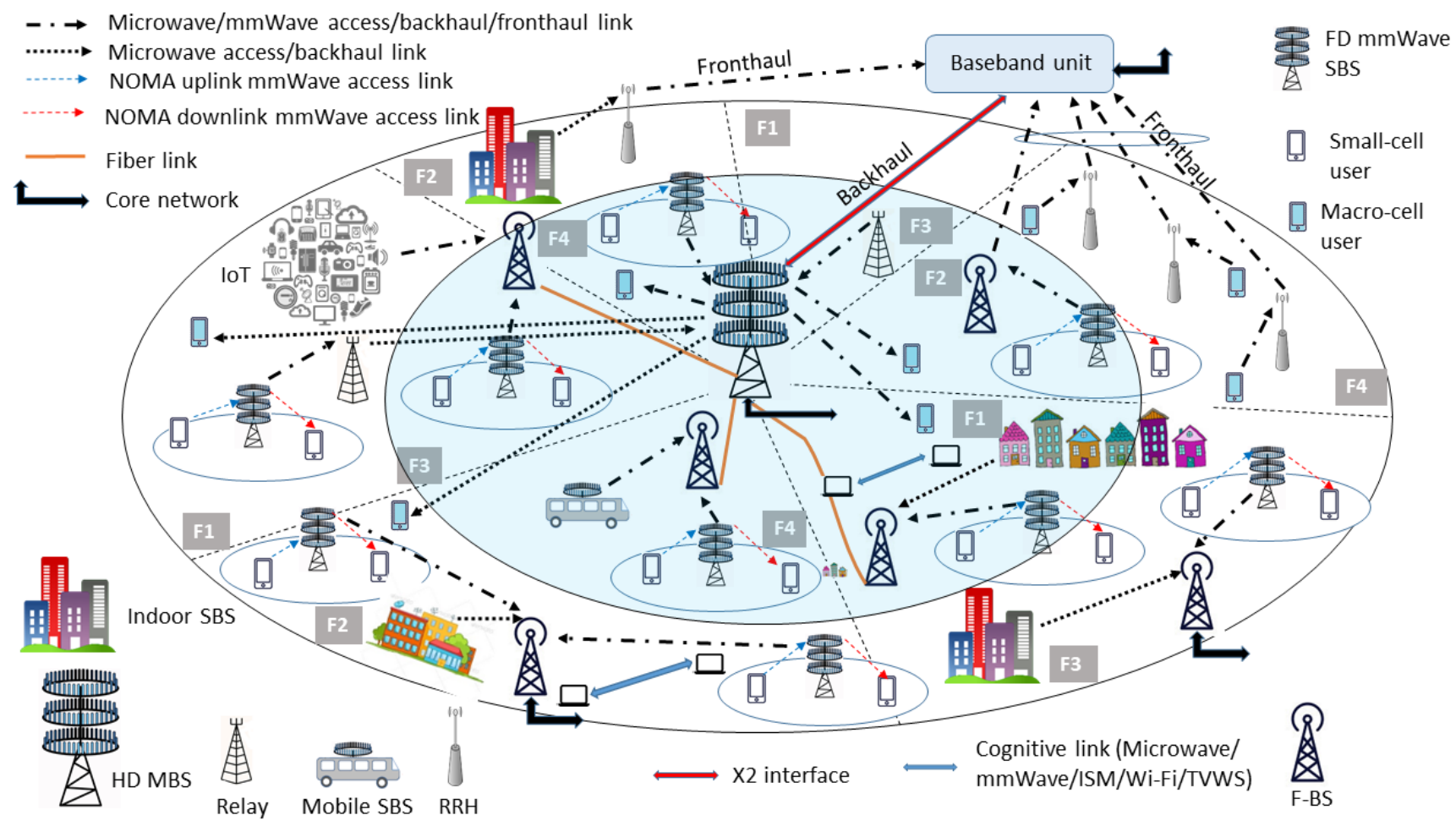

Fig. 1. The wireless access, backhaul and fronthaul links in a macro-cell belonging to an F-RAN architecture; the macro-cell has dense deployment of SCs, and uses technologies like mmWave, mMIMO, NOMA, and FD.

\section{Backhauling in the SCs Network}

The high throughput supported by the access links is infeasible without high capacity backhaul links for the SCs. With the massive increase in the SC deployment, a high capacity and reliable wired optical fiber backhaul connectivity for every $\mathrm{SC}$ is uneconomical and inefficient. Evidently, the wireless backhaul is a suitable solution. However, the development of efficient wireless backhaul solutions is challenging because of the link capacity bottleneck, large network dimensionality, high energy consumption, and interference.

To overcome the challenge of the capacity bottleneck of backhaul links, the spectrum in mmWave bands can provide an appropriate solution. For instance, the SCs, which are in close proximity of the MBS can use the spectrum from the V-and D-bands without much restrictions. The close proximity region is the shaded area in Fig. 1, and is referred to as the closezone. On the other hand, the SCs which are far from the MBS can transmit their backhaul data by using: i) dedicated relays; ii) dedicated F-BSs; iii) the sub- $6 \mathrm{GHz}$, beyond $6 \mathrm{GHz}$ and licensed shared access (SA) spectrum from TV white space (TVWS), radar, and satellite bands. The far region is referred to as the far-zone, which is essentially the region between the boundaries of the close-zone and macro-cell in Fig. 1.

The dedicated relays for backhauling represent a potential solution over deploying the optical fiber cables; however, because of the large network size, this is not efficient in terms of overall capital expenditure and end-to-end delays. Hence, complete dependence on relays is not feasible. Consequently, fixed F-BSs are expected to be deployed within a coverage area; they basically relay the aggregated backhaul traffic from several SBSs to core network either directly or through BBU or MBS. Since the F-BSs are fixed, and less in number compared with the number of SCs, their connectivity to the MBS via optical fiber is viable. Further, the F-BSs can communicate among themselves to exchange information about the serving $\mathrm{SCs}$ for performing coordinated multi-point (CoMP) operations to managing the inter-cell CCI, and thus, improve the network capacity and cell edge user throughput.

The next-generation cellular networks can also employ carrier aggregation (CA) and carrier bonding (CB) techniques for creating a wider bandwidth by using the spectrum from licensed, unlicenced and SA bands. CA techniques can aggregate multiple contiguous and/or non-contiguous carrier components (CCs) belonging to intra- and/or inter-bands. $\mathrm{CB}$ techniques are used to combine contiguous intra-band $\mathrm{CCs}$ in a single wider band. The licensed and SA spectrum available to use is from sub-6 GHz, including TVWS (600-800 $\mathrm{MHz}$ ), radar (960-1400, 2700-3650, 5-5.850 GHz), satellite's $\mathrm{L}$ band, as well as from beyond- $6 \mathrm{GHz}$ including spectrum from mmWave, satellite's (S/C bands), and optical bands. The $\mathrm{CA} / \mathrm{CB}$ in these bands can be enabled through DSA techniques such as sensing, active scanning, and geo-location databases and beaconing. The spectrum from TVWS, radar, and satellite belongs to SA bands, and hence, its usage in the cellular network is performed either via a cognitive underlay or overlay approach. Based on the backhaul link-distance, the MBS can select the appropriate spectrum band for backhaul communication. A distance-based spectrum usage scheme that relies on the CA/CB techniques is shown in Fig. 1. The SBSs 


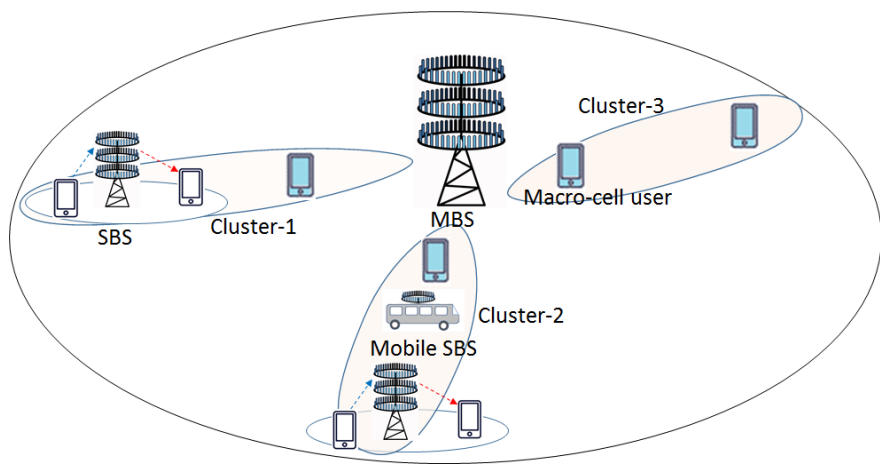

Fig. 2. The MBS serves several SBSs and macro-cell users by grouping them in three clusters.

and relays located in the close-zone tend to use frequencies from mmWave, while they tend to use frequencies from TVWS, radar, and satellite bands when located in the far-zone.

Furthermore, the available spectrum can be utilized efficiently by allowing the SBSs to operate in FD mode, i.e. simultaneous transmission to the users and backhaul transmission to the relay or F-BS or MBS. Additionally, by applying DL power-domain NOMA at the MBS to superimpose the data of two or more SBSs or of macro-cell users and SBSs, improves the backhaul capacity; such a scenario is illustrated in Fig. 2.

\section{OpEn ChaLlenges}

It is certain from the above discussion that the coexistence of all key technologies would significantly improve the capacity of the next generation wireless networks. However, this also brings challenges that cannot be overlooked; some are mentioned as follows:

Interference management: A major challenge is the intraand inter-cell CCI in both mmWave and microwave frequencies. The highly directional LOS beams are essential at mmWave frequencies in the SC for improving the overall cell throughput; however, this decreases the cell-edge user's throughput due to inter-cell CCI. On the other hand, at microwave frequencies, both LOS and NLOS reflected beams create a strong CCI to the users both in and out of the SC. The interference level is more profound in an amorphously deployed UDenseNets. Further, the FD and NOMA links create additional inter-cell CCI and limit the performance. Hence, interference management is critical and plays a vital role in the seamless coexistence of key technologies. The CoMP techniques for interference and coordinated resource management are certainly helpful here; however, they require large amount of CSI. The distributed techniques would provide reasonable solutions with some performance loss.

Joint SBS and RRH selection optimization: In UDenseNets, a user would be in the close proximity of many cells. The SCs in the neighbourhood of a user could be either dominant interferers or strong servers. This depends on the association scheme and the coordination among the neighboring SCs. Furthermore, the performance of the overall network is heavily restricted by the backhaul links. As FD and NOMA techniques enable even more users to be served by an SC, the burden on the backhaul links increases. Hence, it is imperative for each SBS to select an appropriate F-BS or relay. Similarly, for the users associated to RRHs, to select the RRH that has a larger link capacity to the BBU. However, a joint user association to the SBS and/or RRH, and selection of F-BS or relay scheme needs to be investigated; such a solution can further improve the overall network throughput performance.

Green network architecture: Owing to the network densification in the $5 \mathrm{G}$ and beyond networks, the SCs can dynamically adjust their power based on the current needs. To this end, machine learning algorithms are useful as they learn the traffic patterns of individual SC to switch the SBSs on or off. The on/off scheme for the SBSs offers significant improvement in the energy utilization of the network.

\section{Performance Studies}

In this section, we demonstrate the access links throughput gain achieved by implementing FD and NOMA at SCs in an UDenseNet, which operates in mmWave band and employs mMIMO technology. We consider the macro-cell scenario as depicted in Fig. 1. In order to avoid the interference and improve the frequency reuse, the macro-cell area is partitioned into close- and far-zones. Each such zone is further sectored in six sub-regions. Four orthogonal mmWave bands, denoted as F1, F2, F3 and F4, are used in these sub-regions. Since the mmWave signal suffers huge propagation attenuations, the allocation of the set of four mmWave bands shown in Fig. 1 is good enough to avoid any inter-region interference. In simulations, we consider only one such sub-region scenario. In this sub-region, all the SBSs use the same frequency band for access links.

The locations of SCs is modeled via a Poisson point process with density $\lambda_{s}$. The users are uniformly distributed in the macro-cell region. The mmWave channels between the users to the SBSs are modelled as describe in [10]. A summary of simulation parameters and their values is provided in Table III.

TABLE III

SIMULATION PARAMETERS AND THEIR VALUES.

\begin{tabular}{l|c}
\hline Parameter & Values \\
\hline $\begin{array}{l}\text { Transmit power }(\mathrm{dBm}) \\
\text { Noise and residual SI } \\
\text { powers }(\mathrm{dBm})\end{array}$ & $\sigma_{n}^{2}=-104$, and $\sigma_{\mathrm{SI}}^{2}=-110$ \\
$\begin{array}{l}\text { Carrier frequency and sys- } \\
\text { tem bandwidth }\end{array}$ & $f_{c}=28 \mathrm{GHz}$ and $100 \mathrm{MHz}$ \\
$\begin{array}{l}\text { NOMA power coefficients } \\
\text { (weak and strong users) }\end{array}$ & 0.7 and 0.3 \\
$\begin{array}{l}\text { OMA power coefficients } \\
\text { (weak and strong users) }\end{array}$ & 0.5 and 0.5 \\
$\begin{array}{l}\text { Number of transmitting } \\
\text { antennas at SBS and user }\end{array}$ & 64 and 32 \\
$\begin{array}{l}\text { Number of DL and UL } \\
\text { users }\end{array}$ & 2 and 2 \\
$\begin{array}{l}\text { Macro-cell and SC radius } \\
\text { (in meters) }\end{array}$ & 500 and 100 \\
$\begin{array}{l}\text { Path loss (in dB), where } \\
d \geq 1 \text { meter and } c \text { is the } \\
\text { speed of light }\end{array}$ & LOS : $20 \log _{10}\left(4 \pi f_{c} / c\right)+20.1 \log _{10}(d)$ \\
\hline
\end{tabular}

We consider three cases for the SBSs, when they employ: i) OMA and half-duplex (HD) schemes; ii) NOMA and HD 


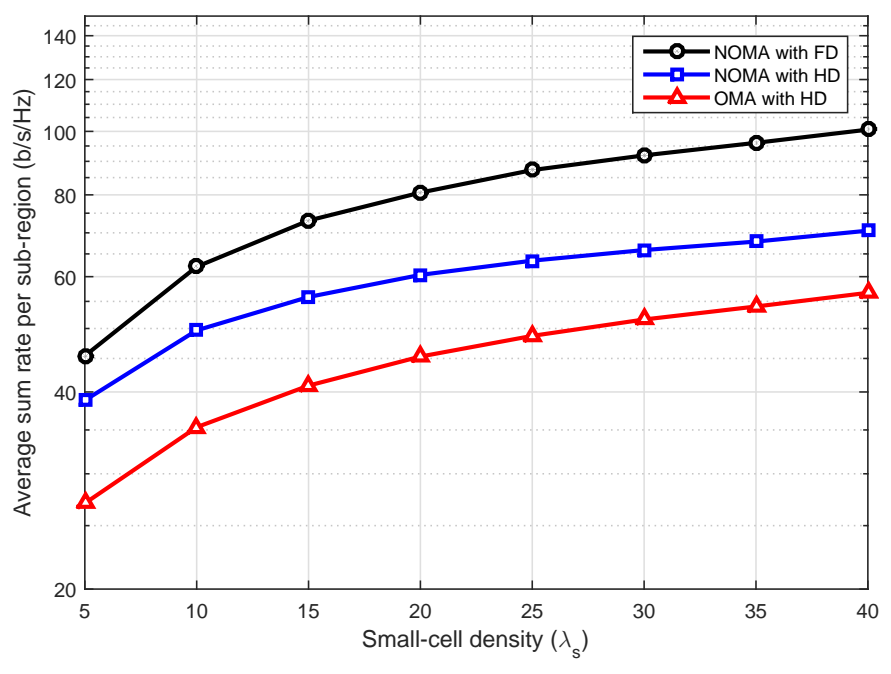

Fig. 3. A comparison of the sum rate of an UDenseNet employing OMA-HD, NOMA-HD and NOMA-FD schemes, respectively.

schemes; and iii) NOMA and FD schemes. The HD and OMA are the traditional technologies, and hence, they are also plotted for gauging the performance over the NOMA and FD technologies. The beamspaced MIMO scheme [14] is employed to reduce the number of RF chains. In each SC, on one hand, two DL users are served by one RF chain by multiplexing them using the NOMA technique in a way similar to the one employed in [10]. On the other hand, two UL users are detected at the SBS using the SuIC technique. The intra- and inter-cell CCIs are considered as noise in the sum rate calculations. Further, we assume that for the FD mode of operation, there are two DL and UL users in each SC scheduled for communication. Contrastingly, for the HD mode of operation, only two DL users are assumed to be scheduled.

Fig. 3 illustrates the average sum rate per sub-region of the network versus the density of SCs for all three cases. The results are obtained for 1000 random topologies. The sum rate is calculated using the Shannon's capacity formula. It can be observed that when both NOMA and FD are employed, a higher sum rate is achieved. In addition, the sum rate in all cases increases with the number of SCs and saturates later on due to high inter-cell CCI.

\section{CONCLUSION}

This article discusses important key enabling technologies, such as network densification, mmWave, mMIMO, NOMA, FD, and DSA, which are identified as solution providers for the capacity crunch problem in $5 \mathrm{G}$ and beyond wireless networks. To date, these technologies have been investigated either alone or in a combination of two or three together. This article envisages the integration of all technologies and discusses the overall benefits and challenges of their coexistence in wireless access and backhaul links. The sum rate of the network is investigated with respect to the increase in the number of SCs, and results show the capacity gains achieved by such coexistence. Lastly, this article paves the way for attaining better network capacity gains if the challenges are addressed efficiently.

\section{REFERENCES}

[1] S. Parkvall et al., "NR: The new 5G radio access technology," IEEE Commun. Standards Mag., vol. 1, no. 4, pp. 24-30, Dec. 2017.

[2] T. Bogale and L. Le, "Massive MIMO and mmWave for $5 \mathrm{G}$ wireless HetNet: Potential benefits and challenges," IEEE Veh. Technol. Mag., vol. 11, no. 1, pp. 64-75, Mar. 2016.

[3] N. Bhushan et al., "Network densification: The dominant theme for wireless evolution into 5G," IEEE Commun. Mag., vol. 52, no. 2, pp. 82-89, Feb. 2014.

[4] T. S. Rappaport et al., "Millimeter wave mobile communications for 5G cellular: It will work!" IEEE Access, vol. 1, pp. 335-349, May 2013.

[5] F. Rusek et al., "Scaling up MIMO: Opportunities and challenges with very large arrays," IEEE Signal Processing Mag., vol. 30, no. 1, pp. 40-60, Jan. 2013.

[6] A. F. Molich et al., "Hybrid beamforming for massive MIMO: A survey," IEEE Commun. Mag., vol. 55, no. 9, pp. 134-141, Sep. 2017.

[7] S. M. Islam et al., "Power-domain non-orthogonal multiple access NOMA in 5G systems: Potentials and challenges," IEEE Cоттип. Surveys Tut., vol. 19, no. 2, pp. 721-742, 2nd Quart., 2017.

[8] G. I. Tsiropoulos et al., "Cooperation in 5G HetNets: Advanced spectrum access and D2D assisted communications," IEEE Wireless Commun. Mag., vol. 24, no. 5, pp. 110-117, Oct. 2017.

[9] W. Feng et al., "When mmWave communications meet network densification: A scalable interference coordination perspective," IEEE J. Select. Areas Commun., vol. 35, no. 7, pp. 1459-1471, Apr. 2017.

[10] B. Wang et al., "Spectrum and energy efficient beamspace MIMONOMA for millimeter-Wave communications using lens antenna array," IEEE J. Select. Areas Commun., vol. 35, no. 10, pp. 2370 - 2382, Oct. 2017.

[11] W. Shin et al., "Non-orthogonal multiple access in multi-cell netwroks: Theory, performance, and practical challenges," IEEE Commun. Mag., vol. 55, no. 10, pp. 176-183, Oct. 2017.

[12] A. Shojaeifard et al., "Massive MIMO-enabled full-duplex cellular networks," IEEE Trans. Commun., vol. PP, no. 99, pp. 1 - 1, Mar. 2017

[13] Y. Sun et al., "Optimal joint power and subcarrier allocation for full-duplex multicarrier non-orthogonal multiple access," IEEE Trans. Commun., vol. 65, no. 3, pp. 1077 - 1091, Mar. 2017.

[14] J. Brady et al., "Beamspace MIMO for millimeter-wave communications: System architecture, modeling, analysis, and measurements," IEEE Trans. Antennas Propagat., vol. 61, no. 7, pp. 3814-3827, Jul. 2015.

[15] M. Zeng et al., "Capacity comparison between MIMO-NOMA and MIMO-OMA with multiple users in a cluster," IEEE J. Select. Areas Commun., vol. 35, no. 10, pp. 2413-2424, Jul. 2017.

\section{BIOGRAPHIES}

ANIMESH YADAV (animeshy@mun.ca) is a Research Associate at Memorial University, Canada. Previously, he worked as a Postdoctoral Research Fellow and Research Scientist at UQAM, Canada, and CWC at University of Oulu, Finland, respectively. During 2003-07, he worked as a Software Specialist at iGate Global Solutions Ltd., India and Elektrobit Oy, Finland. He received the master's and Ph.D. degree from Indian Institute of Technology (IIT) Roorkee, India and University of Oulu, Finland, respectively. He is the recipient of the best paper awards at IEEE WiMOB-2016 and IWCMC-2017. His research interests include enabling technologies for future wireless networks and green communications.

OCTAVia A. DobRe (odobre@mun.ca) is a Professor and Research Chair at Memorial University, Canada. In 2013 she was a Visiting Professor at Massachusetts Institute of Technology, USA, and University of Brest, France. Previously, she was with New Jersey Institute of Technology, USA, and Polytechnic Institute of Technology, Romania. She was the recipient of a Royal Society scholarship and a Fulbright fellowship. Her research interests include enabling technologies for $5 \mathrm{G}$, cognitive radio systems, blind signal identification and parameter estimation techniques, as well as optical and underwater communications. She has authored around 200 referred journal and conference papers in these areas. Dr. Dobre is the EiC of the IEEE Communications Letters. She has served as editor for various prestigious journals, and technical co-chair of different international conferences, such as IEEE ICC and Globecom. She is a member-at-large of the Administrative Committee of the IEEE Instrumentation and Measurement Society and served as chair and co-chair of different technical committees. Dr. Dobre is a Fellow of the Engineering Institute of Canada. 